\title{
Integration of barometric height into RTK Positioning for Fast Ambiguity Refixing
}

\author{
Julius Krause and Patrick Henkel \\ ANavS GmbH \\ Heßstrasse 36, 80798 Munich, Germany \\ julius.krause@anavs.de,patrick.henkel@anavs.de
}

\begin{abstract}
The horizontal positioning accuracy of Global Navigation Satellite System receivers is in general two times higher than the vertical positioning accuracy. The integration of barometric height information improves in particular the vertical positioning accuracy.

In this paper, we integrate differential air pressure measurements into Real-Time Kinematic (RTK) positioning with a Kalman filter. We show that the differential air pressure measurements enable a faster convergence of the float RTK solution and a more reliable ambiguity fixing. Thereby, the proposed method is especially attractive for improving the RTK performance after temporary GNSS outages.
\end{abstract}

Keywords-Positioning, RTK, GNSS, Barometer, Ambiguity Fixing, Kalman Filter

\section{INTRODUCTION}

Real-Time Kinematic (RTK) positioning with Global Navigation Satellite Systems (GNSS) has become very attractive over the last years since it provides centimeter-level positioning accuracies at low cost. The classical RTK positioning is characterized by the following features:

- use of differential measurements between a user and reference station

- use of carrier phase measurements besides pseudorange measurements

- resolution of the integer ambiguities of the periodic carrier phases

The performance of the ambiguity fixing depends essentially on the quality of the much less accurate pseudorange measurements. If the pseudorange measurements are affected by multipath, the ambiguity fixing and re-fixing becomes either slow or unreliable.

In this paper, we integrate differential barometric air pressure information into the RTK positioning to fasten the ambiguity fixing and re-fixing. Fig. 1 visualizes the challenge of ambiguity fixing for RTK positioning: The distance between the parallel wavefronts of a satellite corresponds to the small wavelength of only $19 \mathrm{~cm}$, which is much smaller than the uncertainty of the pseudorange measurements being in the order of several meters. The differential air pressure measurements constrain the height as indicated by the horizontal layer. The intersection of the wavefronts from several satellites, the search space spanned by the pseudorange measurements and the barometric height information results in a position with centimeter-level accuracy.

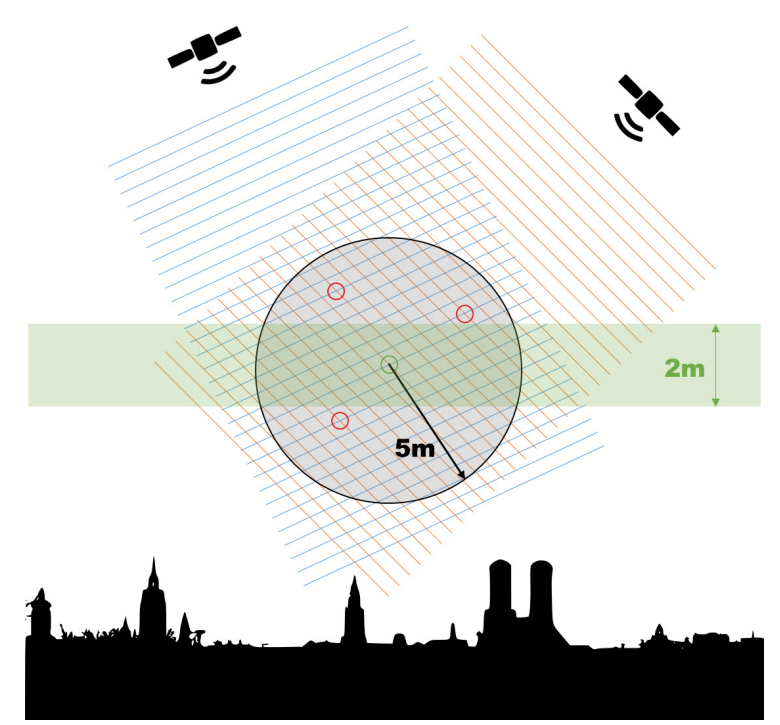

Figure 1. Real-Time Kinematic (RTK) positioning with GNSS pseudorange and carrier phase measurements and barometric air pressure measurements: The GNSS pseudorange measurements are affected by code noise and multipath, which limit the achievable positioning accuracy to a couple of meters (black circle). The GNSS carrier phase measurements are much more accurate but are ambiguous due to the periodicity of the sinusoidal wave (wavefronts). The barometric air pressure measurements provide an independent height information. Both the pseudorange measurements and the air pressure measurements constrain the search space of the phase ambiguities.

\section{MODELING OF BAROMETRIC MEASUREMENTS}

We model the air pressure measurement according to Hopfield [1] as

$$
\begin{aligned}
p_{u}\left(h_{u}\right)= & p_{0}\left(h_{0}\right) \cdot\left(1-\frac{\alpha\left(h_{u}-h_{0}\right)}{T_{0}}\right)^{\gamma} \\
& +\Delta p_{\text {meteo }_{u}}+b_{p_{u}}+\eta_{p_{u}},
\end{aligned}
$$

with the following notations:

$$
\begin{array}{ll}
p_{0}\left(h_{0}\right) & \text { true air pressure } \\
h_{u} & \text { for neutral atmosphere at height } h_{0} \\
T_{0} & \text { height of user } \\
\gamma=\frac{g}{R_{\mathrm{d}} \alpha} & \text { temperature at height } h_{0} \\
g & \text { exponent } \\
R_{\mathrm{d}}=287.1 \frac{\mathrm{J}}{\mathrm{kgK}} & \text { gravitational acceleration } \\
\alpha & \text { specific gas constant } \\
\Delta p_{\text {meteo }_{u}} & \text { temperature lapse rate } \\
& \text { meteorological change of air pressure }
\end{array}
$$


$b_{p_{u}} \quad$ bias of air pressure measurement

$\eta_{p_{u}} \quad$ noise of air pressure measurement

We linearize the first non-linear term of Eq. (1) around the height $h_{0}$ :

$$
\begin{aligned}
& p_{0}\left(h_{0}\right) \cdot\left(1-\frac{\alpha\left(h_{u}-h_{0}\right)}{T_{0}}\right)^{\gamma} \\
& \approx p_{0}\left(h_{0}\right)+\left.\frac{\partial p}{\partial z}\right|_{h_{u}=h_{0}}\left(h_{u}-h_{0}\right)+\mathcal{O}\left(h_{u}^{2}\right),
\end{aligned}
$$

with the partial derivative

$$
\begin{aligned}
\left.\frac{\partial p}{\partial z}\right|_{h_{u}=h_{0}} & =-\left.p_{0}\left(h_{0}\right) \frac{\alpha}{T_{0}} \gamma\left(1-\frac{\alpha\left(h_{u}-h_{0}\right)}{T_{0}}\right)^{\gamma-1}\right|_{h_{u}=h_{0}} \\
& =-\frac{\gamma \alpha}{T_{0}} p_{0}\left(h_{0}\right)=-\frac{g}{R_{\mathrm{d}} T_{0}} p_{0}\left(h_{0}\right)
\end{aligned}
$$

where we used the definition of $\gamma=\frac{g}{R_{\mathrm{d}} \alpha}$ in the last identity.

We perform differential air pressure measurements between two close barometers to eliminate the common meteorological offset, i.e.

$p_{u}\left(h_{u}\right)-p_{r}\left(h_{r}\right) \approx-\frac{g}{R_{\mathrm{d}} T_{0}} p_{0}\left(h_{0}\right)\left(h_{u}-h_{r}\right)+b_{p_{u r}}+\eta_{p_{u r}}$,

with $b_{p_{u r}}$ being the differential barometric sensor bias and $\eta_{p_{u r}}$ being the differential measurement noise.

Fig. 2 shows the air pressure measurements of two close barometers. A drift in the order of $0.5 \mathrm{hPa} /$ hour can be observed due to changing meteorological conditions. The relative offset between both measurements is much more stable and caused by different sensor biases.

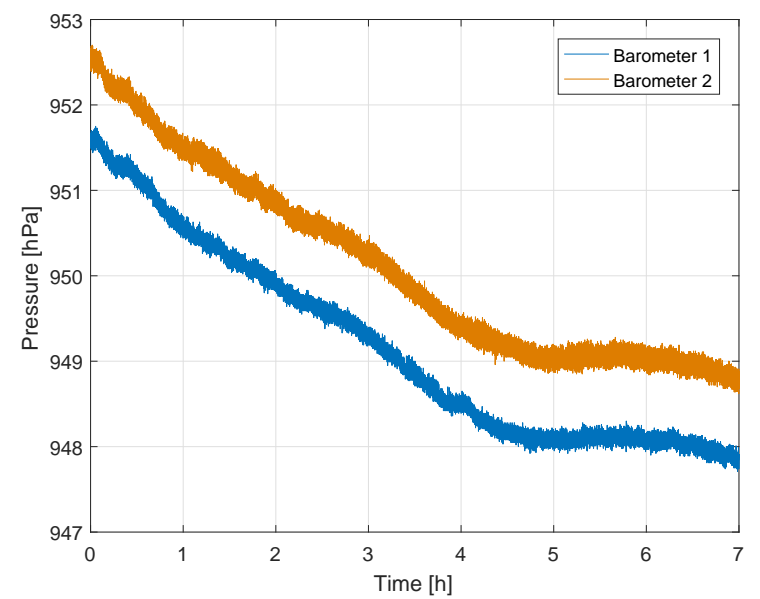

Figure 2. Air pressure measurements of two close barometers: Both barometers show a drift in the order of $0.5 \mathrm{hPa}$ / hour due to changing meteorological conditions. The relative offset between both measurements is caused by different sensor biases and is much more stable than the change in meteorological conditions.

\section{INTEGRATION OF BAROMETRIC HEIGHT INTO RTK POSITIONING}

\section{A. Float Solution}

In this section, we describe the integration of barometric air pressure measurements in the float RTK positioning.

We start by a brief review of the models for double difference (DD) GNSS measurements: The DD carrier phase measurements of receiver pair $u$ and $r$ and satellite pair $k$ and $l$ are modeled according to [3] as

$$
\begin{aligned}
\lambda \varphi_{u r}^{k l} & :=\lambda\left(\varphi_{u}^{k}-\varphi_{r}^{k}\right)-\lambda\left(\varphi_{u}^{l}-\varphi_{r}^{l}\right) \\
& =\left(\vec{e}_{u}^{k l}\right)^{\mathrm{T}} \vec{x}_{u r}+\lambda N_{u r}^{k l}+\varepsilon_{u r}^{k l}
\end{aligned}
$$

with the following notations:

$\lambda$
$\varphi_{u}^{k}$
$\vec{x}_{u}$
$\vec{x}_{u r}$
$\vec{x}^{k}$
$\vec{e}_{u}^{k}=\frac{\vec{x}_{u}-\vec{x}^{k}}{\left\|\vec{x}_{u}-\vec{x}^{k}\right\|}$

$\vec{e}_{u}^{k l}=\vec{e}_{u}^{k}-\vec{e}_{u}^{l}$

$N_{u r}^{k l}=N_{u r}^{k}-N_{u r}^{l}$

$\varepsilon_{u r}^{k l}=\varepsilon_{u r}^{k}-\varepsilon_{u r}^{l}$ wavelength of GNSS carrier phase carrier phase as tracked by Phase Locked Loop (PLL) absolute position of GNSS receiver relative position

between $u$-th and $r$-th GNSS receiver absolute position of GNSS satellite line of sight vector pointing from satellite to receiver differential line of sight vector DD integer ambiguity DD phase noise
The DD pseudorange measurements are defined similarly:

$$
\begin{aligned}
\rho_{u r}^{k l} & :=\left(\rho_{u}^{k}-\rho_{r}^{k}\right)-\left(\rho_{u}^{l}-\rho_{r}^{l}\right) \\
& =\left(\vec{e}_{u}^{k l}\right)^{\mathrm{T}} \vec{x}_{u r}+\Delta \rho_{\mathrm{MP}, u r}^{k l}+\eta_{u r}^{k l},
\end{aligned}
$$

with the DD pseudorange multipath error $\Delta \rho_{\mathrm{MP}, u r}^{k l}$ and the DD pseudorange measurement noise $\eta_{u r}^{k l}$.

We stack the DD carrier phase and pseudorange measurements of all satellites and the differential air pressure measurement at epoch $n$ in a single measurement vector:

$$
z_{n}=\left(\lambda \varphi_{u r}^{1 l}, \ldots, \lambda \varphi_{u r}^{K l}, \rho_{u r}^{1 l}, \ldots, \rho_{u r}^{K l}, p_{u}\left(h_{u}\right)-p_{r}\left(h_{r}\right)\right)_{n}^{\mathrm{T}} .
$$

We assume that the GNSS receiver and barometer are colocated at both the user location and reference location. Thereby, both sensors include an information on the height component of the relative position $\vec{x}_{u r}$. We additionally assume that the differential barometric measurements are calibrated, i.e. the differential bias $b_{p_{u r}}$ is known with sufficient accuracy and can be removed respectively.

Thus, the stacked DD carrier phase, pseudorange and differential air pressure measurements can be expressed in terms of the following unknown parameter set:

$$
x_{n}=\left(\left(\vec{x}_{u r}\right)^{\mathrm{T}}, N_{u r}^{1 l}, \ldots, N_{u r}^{K l}, \Delta \rho_{\mathrm{MP}, u r}^{1 l}, \ldots, \Delta \rho_{\mathrm{MP}, u r}^{K l}\right)^{\mathrm{T}} .
$$

The measurement models of Eq. (4), (5) and (6) show a linear relationship between the measurements and state parameters:

$$
z_{n}=H_{n} x_{n}+\eta_{z_{n}},
$$


where the mapping matrix $H_{n}$ is implicitly defined by the measurement models, and $\eta_{z_{n}} \sim \mathcal{N}\left(0, \Sigma_{z_{n}}\right)$ denotes the measurement noise. The last row of $H_{n}$ refers to the differential barometric measurements. The respective matrix elements of $H_{n}$ are given by

$$
\left(H_{n}\right)_{2 K+1, j}=\left\{\begin{array}{c}
-\frac{g}{R_{\mathrm{d}} T_{0}} p_{0}\left(h_{0}\right) \\
0
\end{array}\right\} \text { for } \quad\left\{\begin{array}{l}
j=3 \\
j \neq 3
\end{array}\right\} .
$$

The time-behaviour of the state parameters of Eq. (8) is described with sufficient accuracy by a linear state space model (see also [3]) given by

$$
x_{n}=\Phi_{n} x_{n-1}+\eta_{x_{n}},
$$

with the state transition matrix $\Phi_{n}$, the state vector $x_{n-1}$ of the previous epoch, and the process noise $\eta_{x_{n}} \sim \mathcal{N}\left(0, \Sigma_{x_{n}}\right)$.

We use a standard Kalman filter as described in [4] for estimating the state parameters. The Kalman filter includes a state prediction using the state space model of Eq. (11), i.e.

$$
\hat{x}_{n}^{-}=\Phi_{n} \hat{x}_{n-1}^{+},
$$

where $\hat{x}_{n-1}^{+}$denotes the a posteriori state estimate of the previous epoch. The covariance matrix of the predicted state follows from Eq. (12) as

$$
\Sigma_{\hat{x}_{n}^{-}}=\Phi_{n} \Sigma_{\hat{x}_{n-1}^{+}} \Phi_{n}^{\mathrm{T}}+\Sigma_{x_{n}} .
$$

The state prediction is updated with the measurements $z_{n}$ :

$$
\hat{x}_{n}^{+}=\hat{x}_{n}^{-}+K_{n}\left(z_{n}-H_{n} \hat{x}_{n}^{-}\right),
$$

where the Kalman gain $K_{n}$ is determined such that the trace of the state update covariance matrix is minimized.

\section{B. Ambiguity Fixing}

In this section, we describe the integration of barometric information in the ambiguity fixing.

The float ambiguity estimates $\hat{N}$ and their covariance matrix $\Sigma_{\hat{N}}$ are the basis for ambiguity fixing. We search the integer ambiguity candidate vectors within a certain search space volume $\chi^{2}$ centered at the float solution, i.e.

$$
\|\hat{N}-N\|_{\Sigma_{\hat{N}}^{-1}}^{2} \stackrel{!}{\leq} \chi^{2}
$$

The sum of squared ambiguity residuals can be expressed in terms of conditional ambiguity estimates as (see Teunissen [2]):

$$
\|\hat{N}-N\|_{\Sigma_{\hat{N}}^{-1}}^{2}=\frac{\left(N^{k}-\hat{N}^{k \mid 1, \ldots, k-1}\right)^{2}}{\sigma_{\hat{N} k \mid 1, \ldots, k-1}^{2}},
$$

with the $k$-th conditional ambiguity estimate $\hat{N}^{k \mid 1, \ldots, k-1}$ and its variance $\sigma_{\hat{N}^{k \mid 1, \ldots, k-1}}^{2}$. We plug Eq. (16) into the inequality (15) to obtain:

$$
\begin{aligned}
\frac{\left(N^{k}-\hat{N}^{k \mid 1, \ldots, k-1}\right)^{2}}{\sigma_{\hat{N}^{k \mid 1, \ldots, k-1}}^{2}} & \leq \chi^{2}-\sum_{l=1, l \neq k}^{K} \frac{\left(N^{l}-\hat{N}^{l \mid 1, \ldots, l-1}\right)^{2}}{\sigma_{\hat{N}^{l \mid 1, \ldots, l-1}}^{2}} \\
& \leq \chi^{2}-\sum_{l=1}^{k-1} \frac{\left(N^{l}-\hat{N}^{l \mid 1, \ldots, l-1}\right)^{2}}{\sigma_{\hat{N}^{l \mid 1, \ldots, l-1}}^{2}}
\end{aligned}
$$

Solving this inequality for $N^{k}$ results in two bounds: The upper bound is given by

$$
N^{k} \leq \hat{N}^{k \mid 1, \ldots, k-1}+\sigma_{\hat{N}^{k \mid 1, \ldots, k-1}} \sqrt{\bar{A}},
$$

and the lower bound is given by

$$
N^{k} \geq \hat{N}^{k \mid 1, \ldots, k-1}-\sigma_{\hat{N}^{k \mid 1, \ldots, k-1}} \sqrt{A},
$$

with

$$
A=\chi^{2}-\sum_{l=1, l \neq k}^{K} \frac{\left(N^{l}-\hat{N}^{l \mid 1, \ldots, l-1}\right)^{2}}{\sigma_{\hat{N}^{l \mid 1, \ldots, l-1}}^{2}}
$$

The barometric height information is used to exclude all inconsistent ambiguity candidates and, thereby, to reduce the number of candidates and branches of the tree search. The reduced number of branches substantially improves the reliability and efficiency of the tree search at the price of the additional height consistency check.

\section{Measurement Results}

The proposed method was tested with two ANavS MultiSensor modules including a Multi-GNSS receiver, a 3D accelerometer, a 3D gyroscope, a 3D magnetometer, a barometer and a thermometer. The measurements were taken at a location with good satellite visibility. Temporary GNSS outages were generated by putting a metallic plate over the GNSS antennas.

Fig. 3 shows the fixed phase residuals of the tracked GPS satellites during epochs with good satellite visibility as well as during epochs without any GNSS signal reception. The latter one leads to gaps and/ or an increased magnitude of the phase residuals. Once the satellite visibility improves, the float solution converges again to a more stable solution.

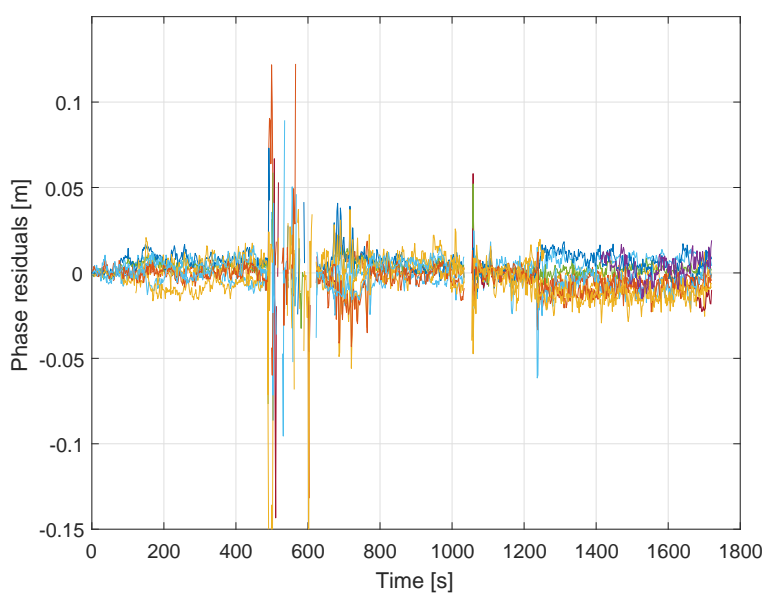

Figure 3. Residuals of RTK positioning with temporary GNSS outages: The residuals are in the order of $1 \mathrm{~cm}$ during good satellite visibility, and increase to $10 \mathrm{~cm}$ and more during GNSS outages.

Fig. 4 shows the benefit of barometric aiding for the convergence of the float RTK solution after three temporary GNSS outages. We can observe that the barometric height enables a much faster convergence than the unaided baseline estimation. We would like to emphasize that the barometric height 
supports the convergence of both the vertical and horizontal baseline components since all estimated state parameters are correlated.
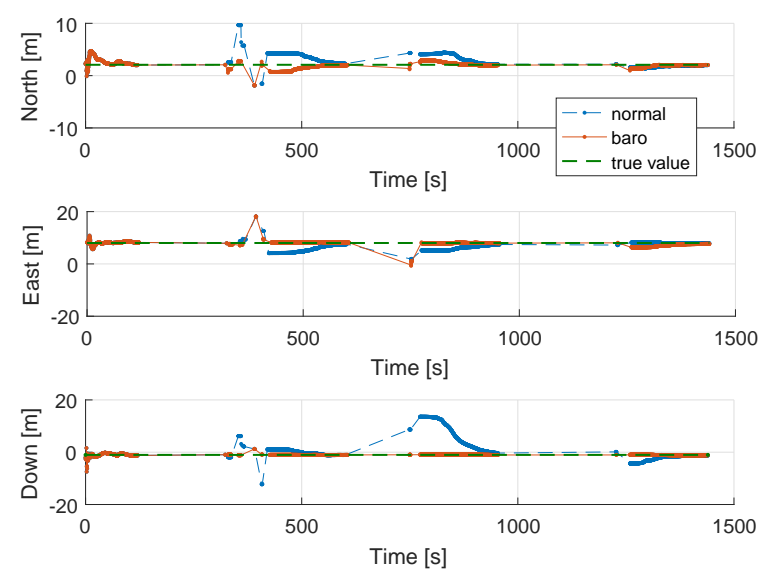

Figure 4. Benefit of barometric aiding for float RTK solution: The barometric height enables a much faster convergence and higher accuracy for the baseline down and north components.

Fig. 5 provides an enlarged view of the estimated baseline north and down components. Obviously, the estimated down component is much more accurate with barometric aiding than without barometric aiding. However, the barometric aiding also reduces the errors in the estimated north component especially in the first epochs after each re-start.

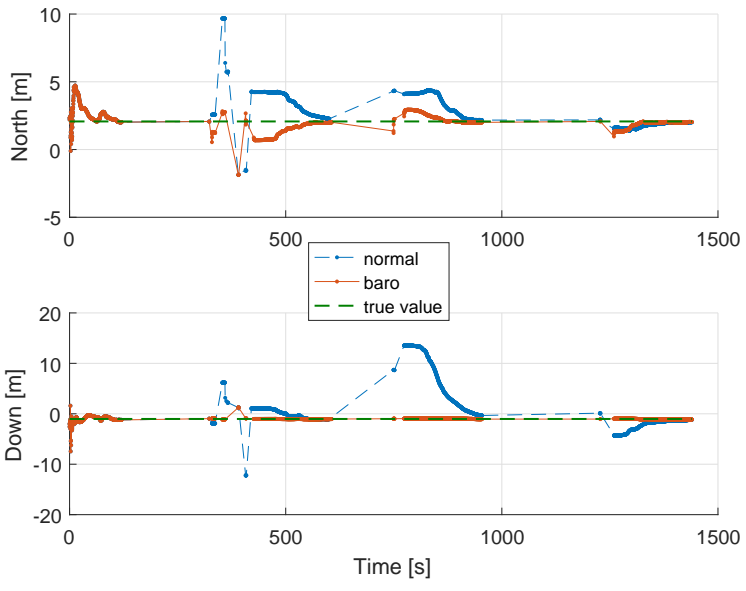

Figure 5. Enlarged view of the estimated baseline north and down components from Fig. 4: The barometric height enables a much faster convergence and higher accuracy for the baseline down and north components.

Fig. 6 shows the benefit of the barometric aiding for RTK ambiguity fixing by comparing the estimated baseline down components with and without barometric aiding: The initial standard RTK fix (without barometric measurements) is shown in the left (blue dashed line with dots). The first standard ambiguity re-fix (shown in black with stars) without barometric aiding results in a consistent solution with an error of only 1 $\mathrm{cm}$. The second standard re-fix without barometric aiding is not shown since it resulted in a wrong ambiguity fixing with an error in the height component of more than 1 meter. The barometric aiding prevents these severely-biased re-fixings, i.e. both the 1st re-fix (dashed orange line with dots) and 2nd re-fix (solid yellow line with dots) with barometric aiding lead to correctly fixed solutions. The only disadvantage of the barometric aiding is that the 1 st re-fix is achieved slightly later as the barometric measurements need to be filtered to some extent to reduce the inherent noise level.

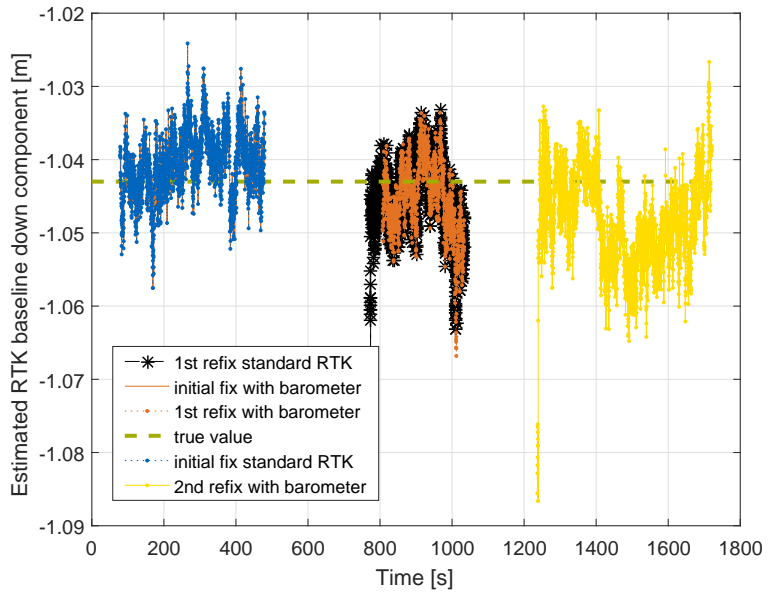

Figure 6. Performance of RTK positioning with and without barometric aiding: The use of a calibrated barometer enables two consistent re-fixes with centimeter-level accuracy, while the unaided solution achieves only one correct re-fix and one completely wrong re-fix. The latter one is not shown since the error in the height component is 100 times larger than the error of correct ambiguity re-fixes.

\section{CONCLUSiON}

In this paper, we described the integration of differential barometric air pressure measurements into Real-Time Kinematic (RTK) positioning with GNSS carrier phase and pseudorange measurements. The barometric measurements were included in both the float RTK solution and the actual ambiguity fixing. The measurement results show a faster convergence of the float solution and a more reliable ambiguity fixing.

The proposed method is in particular attractive for improving the RTK positioning after temporary GNSS outages, e.g. after driving below a bridge.

\section{REFERENCES}

[1] H.S. Hopfield, Two-Quartic Tropospheric Refractivity Profile for Correcting Satellite Data, Journal of Geophysical Research (JGR), vol. 74, pp. 4487 - 4499, 1969.

[2] P.J.G. Teunissen, The Least-Squares Ambiguity Decorrelation Adjustment - A method for fast GPS ambiguity fixing, Journal of Geodesy, vol. 70, pp. $65-82,1995$.

[3] P. Henkel and A. Sperl, Precise RTK Positioning with GPS/ INS Tight Coupling and Multipath Estimation, Proc. of ION Int. Techn. Meet., Monterey, CA, USA, pp. 1015 - 1023, Jan. 2016.

[4] R.G. Brown and P.Y.C. Hwang, Introduction to Random Signals and Applied Kalman Filtering, John Wiley and Sons, 4th edition, 2012. 